\title{
Effects of topical Vancomycin Dressing on Methicillin-Resistant Staphylococcus Aureus (MRSA) positive diabetic foot ulcers
}

\author{
Anas Bin Saif ${ }^{1}$, Sohail Jabbar ${ }^{2}$, Muhammad Saeed Akhtar ${ }^{3}$, \\ Ahmed Mushtaq 4 , Mansoor Tariq ${ }^{5}$
}

\begin{abstract}
Objective: To compare the effects of simple saline dressings versus topical vancomycin dressings on Methicillin-resistant Staphylococcus Aureus positive chronic diabetic foot ulcers.

Methods: It was quasi experimental study conducted in Combined Military Hospital Kohat and PNS-Shifa Hospital Karachi from 01 January 2017 to 31 December 2017. A total of 23 patients were included based on non-probability convenient sampling who had diabetes and presented with foot ulcers for more than two weeks showing positive growth of Methicillin-Resistant Staphylococcus Aureus. The patients were treated with simple saline soaked dressings and debridement at first for three weeks followed by three weeks of topical vancomycin dressings with debridement. Thus patients served as their own controls

Results: The average change in surface area with saline dressing was $+1.73 \pm 1.53 \mathrm{~cm}^{2}$ per week whereas with vancomycin soaked dressing it was $-0.06 \pm 1.60 \mathrm{~cm}^{2}$ per week $(p<0.05)$. The average exudate also decreased from $1.78 \pm 1.23$ to $0.99 \pm 0.72(p<0.05)$ and same trend was observed in percentage of slough covering the ulcer from $45 \% \pm 22.3 \%$ to $24.3 \% \pm 12.90 \%(p<0.05)$ with vancomycin dressing. Moreover, fifteen patients had negative culture for MRSA within 2 weeks.

Conclusion: Vancomycin impregnated dressing in MRSA positive Diabetic foot may help achieve early healing as compared to simple conventional dressings with no systemic toxicity.
\end{abstract}

KEYWORDS: Diabetic foot ulcer, MRSA, Vancomycin.

doi: https://doi.org/10.12669/pjms.35.4.368

How to cite this:

Saif AB, Jabbar S, Akhtar MS, Mushtaq A, Tariq M. Effects of topical Vancomycin Dressing on Methicillin-Resistant Staphylococcus Aureus (MRSA) positive diabetic foot ulcers. Pak J Med Sci. 2019;35(4):1099-1103.

doi: https://doi.org/10.12669/pjms.35.4.368

This is an Open Access article distributed under the terms of the Creative Commons Attribution License (http://creativecommons.org/licenses/by/3.0), which permits unrestricted use, distribution, and reproduction in any medium, provided the original work is properly cited.

1. Dr. Anas Bin Saif, MBBS, FCPS

Consultant General Surgeon,

2. Dr. Sohail Jabbar, MBBS, FCPS

Consultant General Surgeon,

3. Dr. Muhammad Saeed Akhtar, MBBS, FCPS, FACS

Consultant General and Laparoscopic Surgeon,

HoD Surgical Department,

4. Dr. Ahmed Mushtaq, MBBS, FCPS , OJT

Consultant General and Orthopedic Surgeon,

5. Dr. Mansoor Tariq, MBBS, FCPS

Consultant General Surgeon,

1-5: Department of Surgery,

Combined Military Hospital, Kohat, Pakistan.

Correspondence:

Dr. Anas Bin Saif,

Department of Surgery,

Combined Military Hospital,

Kohat, Pakistan.

Email: anasbinsaif02@gmail.com

* Received for Publication:

January 12, 2019

* Revision Received:

* Revision Accepted:

\section{INTRODUCTION}

Globally it is estimated that 382 million people suffer from diabetes with a prevalence of $8.3 \%$. In Pakistan prevalence of this devastating disease is $6.8 \%{ }^{1}$ Diabetic foot is defined as a foot affected by ulceration that is associated with neuropathy and/ or peripheral arterial disease of the lower limb in a patient with diabetes. Diabetic foot infections range in severity from superficial paronychia to deep infection involving bone. Types of infection include cellulitis, myositis, abscesses, necrotizing fasciitis, septic arthritis, tendinitis, and osteomyelitis. It is estimated that about $5 \%$ of all patients with diabetes present with a history of foot ulceration, while the lifetime risk of diabetic patients developing this complication is $15 \% .^{2,3}$ Foot ulceration and infection are the leading risk factors for amputation. ${ }^{4}$ This 
puts a great emphasis on early and accurate management of diabetic foot ulcer. Treatment of diabetic foot involves a multimodal approach including debridement of the wound, management of any infection, revascularization procedures when indicated, glycemic control and off-loading of the ulcer. Other methods have also been suggested to be beneficial as add-on therapies, such as hyperbaric oxygen therapy, use of advanced wound care products, and negative-pressure wound therapy (NPWT).

Debridement is one of the gold standards in wound healing management, significantly contributing to the healing process of the wound, including the diabetic ulcer. ${ }^{5}$ Available data on the use of dressings in diabetic wounds is limited still there is evidence of their role in prevention of infection and enhancement of wound healing. ${ }^{6,7}$

Most ulcers have multi-microbial growth, however, Staphylococcus Aureus is most common. Methicillin Resistant Staphylococcus Aureus (MRSA) also possess a serious threat to Diabetic foot ulcers (DFU) as 30-50\% ulcers have MRSA positive strain. ${ }^{8}$ Studies done in our country shows that burden of disease is very high in our setup due to poor glycemic control and lack of awareness. A large number of patients present with high grade diabetic wound in our clinics. ${ }^{9,10}$

Therefore, management of diabetic foot ulcers remains a major therapeutic challenge for our surgeons especially MRSA positive DFUs, which implies an urgent need to review strategies and treatments in order to achieve the goals and reduce the burden of care in an efficient and cost-effective way. So we designed this study to see effects of topical vancomycin dressing on MRSA positive diabetic foot ulcers.

\section{METHODS}

After ethical approval from ethical committee of hospital this quasi experimental study was planned from 01 Jan 2017 to 31 Dec 2017. All the patients suffering from diabetic foot ulcer for more than 02 weeks and having MRSA positive strain were included. Patients suffering from sepsis, osteomyelitis, allergic to vancomycin and those who did not gave written informed consent were excluded from the study. The data was collected by non-probability convenient sampling technique. The patients were treated at first with saline dressings and debridement twice weekly for first three weeks then the same wound was treated with vancomycin impregnated dressing for next three weeks. ${ }^{11,12}$ Thus patients served as their own controls. Vancomycin dressing was made by mixing $500 \mathrm{mg}$ of injection in $5 \mathrm{ml}$ Saline and spraying it on dry gauze placed in wound. The patients were not given any parenteral or oral antibiotics.

The variables measured apart from demographic data were change in surface area, amount of exudate and amount of slough covering the wound. Area of wound was calculated by linear method, multiplying greatest length to greatest width in centimeters after sharp debridement. Area was calculated at the end of week subtracting it from previous calculated area to get the change in surface area and mean change in area was calculated for simple dressing at end of third week by adding the change in area per week and dividing it by 3 . Similar technique was applied for vancomycin dressing. The exudate was assessed by scoring system where $0=$ none, $1=$ scanty, $2=$ some or minimal, $3=$ moderate and $4=$ frank pus. ${ }^{11}$ SPSS Inc. version 17 was used for data analysis. Mean and Standard deviation was calculated for each variable. Paired t-test was applied to calculate the p-value. Value less than 0.05 was taken as significant keeping confidence interval of $95 \%$.

\section{RESULTS}

A total number of 23 patients were included in the study. There were $15(65 \%)$ males and $8(35 \%)$ female subjects. Average age of males was $59 \pm 5.78$ years and females was $55 \pm 4.66$ years (cumulative age was $57 \pm 7.72$ years). Average time for which patients had diabetes was $10 \pm 4.5$ years. At the time of presentation $22(95 \%)$ patients had peripheral neuropathy, $14(61 \%)$ had peripheral vascular disease, 10 (43\%) had other co-morbids and 5 (22\%) had some form of malnutrition.

The average change in size per week is shown in Fig.1. There was a net increase in surface area of

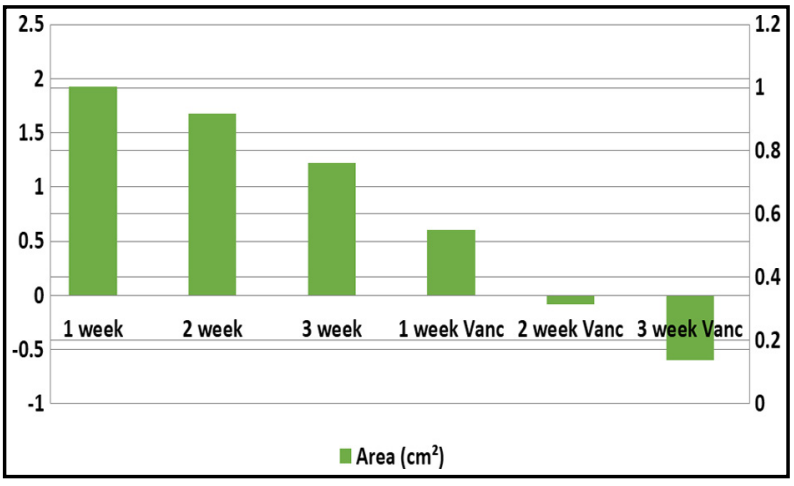

Fig.1: Bar graph showing average change in surface area per week. 


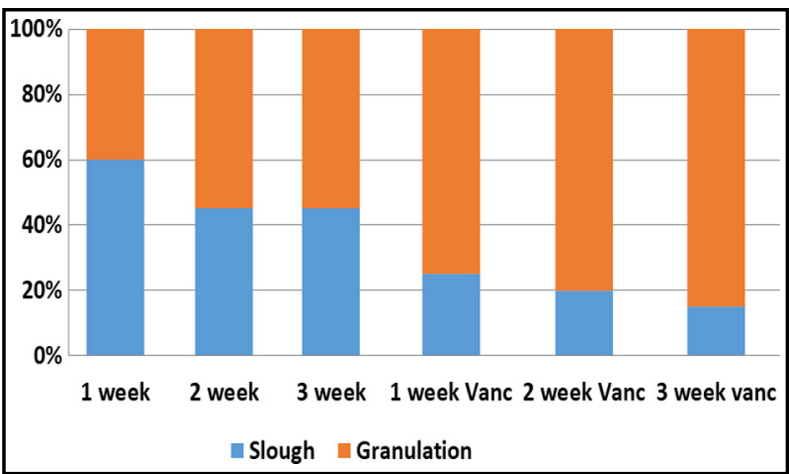

Fig.2: Bar graph showing percentage of slough and granulation per week.

$1.93 \mathrm{~cm}^{2}$ in first week followed by 1.68 and $1.22 \mathrm{~cm}^{2}$. However, after third week, when vancomycin was added to dressings, the surface area of wounds started to decrease from average $0.6 \mathrm{~cm}^{2}$ to -0.08 $\mathrm{cm}^{2}$ and $0.6 \mathrm{~cm}^{2}$ in last week. The net change in amount of slough covering the surface of wound is shown in Fig.2. There was net decrease of $15 \%$ slough using saline dressing in first three weeks, whereas, with vancomycin dressings the percentage granulation increased by $30 \%$ in next three weeks. Similarly, the amount of exudate was objectively assessed and the exudate at end $3^{\text {rd }}$ week was $1.78 \pm 1.23$ with use of saline dressing and when vancomycin was added in the following 3 weeks the average exudate was decreased to $0.99 \pm 0.72$. The comparison in terms of change in surface area, amount of exudate and percentage of slough covering the wound between simple dressings and vancomycin dressings is shown in Table-I. There is a significant statistical difference between the two groups in terms of these variables depicted by p-value <0.05. Moreover 15 (65\%) patients had negative cultures after 2 weeks of using vancomycin dressing. There was no systemic toxicity or allergy reported in all cases.

\section{DISCUSSION}

Infections especially of foot in diabetic patients can have serious outcomes if not managed properly. Another emerging serious concern is development of resistance to antibiotics especially in chronic wounds as DFU. ${ }^{13}$ Our study showed a cumulative age of $57 \pm 7.72$ years and the duration of diabetes was $10 \pm 4.5$ years at which the patients develop DFU. The age and duration at which patients of diabetes develop DFU is less as compared to international studies. ${ }^{14}$ Globally foot ulcers are common in diabetics with age over 60 years and having diabetes for 12 or more years. ${ }^{15}$ Relatively

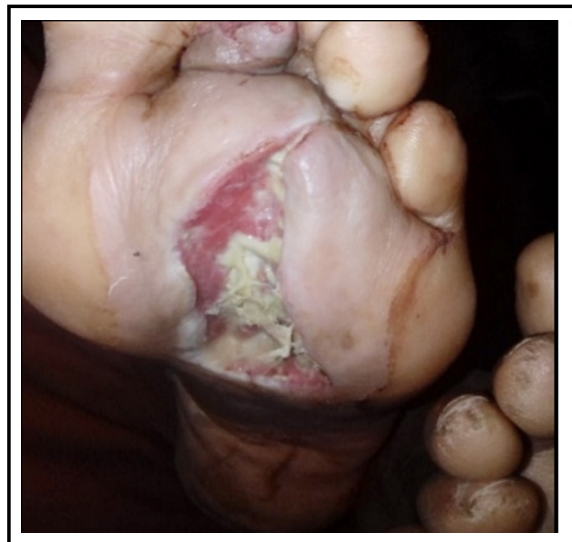

Ulcer after 3 weeks Conventional dressing

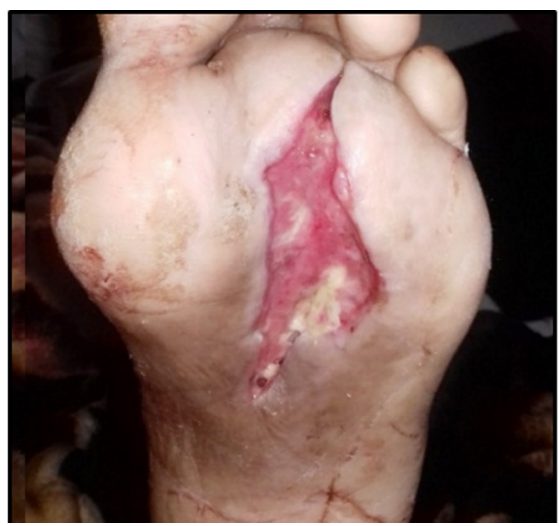

Ulcer after 4 weeks 1 week Vanc use

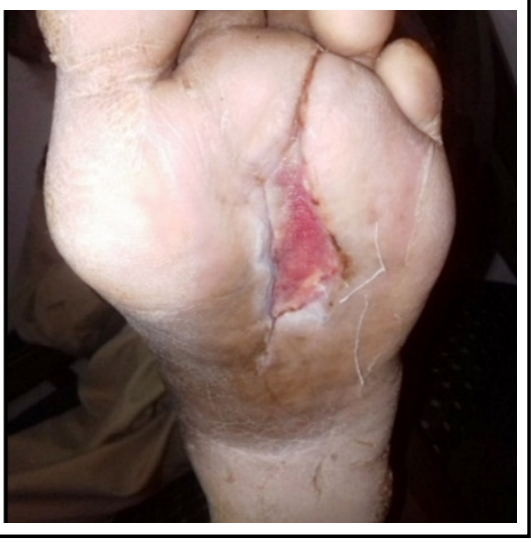

Ulcer after 6 weeks 3 week Vanc use

Fig.3: Showing change in surface area in 63 years old male patient.

Table-I: Showing the change in surface area, amount of exudate and percentage slough comparison between two groups.

\begin{tabular}{clccc}
\hline S. No. & Variable & Simple Dressing & Vancomycin dressing & $\begin{array}{c}p \text {-Value } \\
\text { (paired t-test) }\end{array}$ \\
\hline 1 & Change in surface area & $+1.73 \pm 1.53 \mathrm{~cm}^{2}$ & $-0.06 \pm 1.60 \mathrm{~cm}^{2}$ & $<0.05$ \\
2 & Amount of exudate & $1.78 \pm 1.23$ & $0.99 \pm 0.72$ & $<0.05$ \\
3 & Percentage of slough & $45 \%( \pm 22.3 \%)$ & $24.3 \%( \pm 12.90 \%)$ & $<0.05$ \\
\hline
\end{tabular}


young age and early development of DFUs in our population can be due to lack of awareness and lack of access to better diagnostic facilities.

The most common cause of DFU in our study was neuropathy followed by peripheral arterial disease which is consistent with internationally published studies. ${ }^{16}$ Our study showed that male diabetic patients develop DFU more commonly (65\% males). The study conducted by Amjad SS and colleagues showed that male to female ratio for DFUs was 2:1 which is consistent with our study. ${ }^{17}$ The male preponderance can be due to mal-foot ware and frequent out door traveling. In our study the MRSA positive strain was present in $20 \%$ of all DFUs with Staphylococcus Aureus as most common organism isolated in $55 \%$ of cases. In Pakistan, MRSA positive strains have been found in $20-40 \%$ of DFUs $^{18}$ whereas internationally 30 $50 \%$ of patients with DFU have MRSA strain. ${ }^{5}$ The most common single organism isolated from DFUs is Staphylococcus Aureus which was also observed in our case. ${ }^{19}$

This is the first ever study conducted in Pakistan to encompass the treatment of DFU in MRSA positive strains. Various antibiotics have been studied for topical use in DFUs as metronidazole, neomycin, polymyxin B but vancomycin has not been studied extensively. ${ }^{20,21}$ Our findings depicted that there was significant decrease in exudate and average change in surface area by application of vancomycin impregnated dressings as compared to simple dressing ( $\mathrm{p}$-value <0.01). There was also a rapid decline in the percentage of slough in wound with simultaneous increase in granulation tissue which almost doubled from $15 \%$ to $30 \%$ (p-value $<0.01)$ when vancomycin dressing was used, pointing to the fact that topical vancomycin may help in early wound healing. Moreover, the bacterial culture was also negative for MRSA after two weeks of application in $65 \%$ cases. There was also no hypersensitivity reported in our study with vancomycin. A randomized controlled trial of 426 patients showed that triple antibiotic topical application significantly reduces infection rate in minor wounds. ${ }^{21}$ Albaugh KW et al. studied the effects of vancomycin on chronic wounds and found out that topical applications reduce the bacterial count and may promote healing which was also found in our study. ${ }^{11}$ Whereas Simons et al showed no role of topical antibiotics in severe wound infections in head and neck surgeries. ${ }^{22}$ There are many advantages of using topical antibiotics as high and sustainable concentration of antibiotic available at site of infection, limited total amount of antibiotic needed, limited possibility for systemic toxicity, may prevent development of resistance as systemic drug is not given and it can be applied as OPD case. However, there are some disadvantages as well like minimal penetration to surrounding tissue limits its ability to be used in more severe infection, only few preparations are available, there is possible risk of allergic reaction and there is risk of possible alteration of normal skin flora. ${ }^{21}$ Since the patients were not given any parental or enteral antibiotics so it is worthwhile to apply topical antibiotic application while avoiding systemic use.

There were a few limitations in our study. Sample size is small and data was compared of only one part of country so its result cannot be generalized to whole population. Moreover, the patients were serving as their own controls and were treated with saline dressings first so there is a possibility of procedural bias. Moreover, the cost effect relationship of use of vancomycin was not studied. The author recommends a multi-center randomized controlled trial to evaluate the efficacy of topical vancomycin use is MRSA positive strains.

\section{CONCLUSION}

The use topical preparation of vancomycin in MRSA positive strains in chronic diabetic foot wounds help in significantly reducing the average surface area, amount of exudate and percentage of slough covering the wound as compared to conventional saline soaked dressings. It also simultaneously causes significant decrease in positive MRSA cultures and may promote early healing. The systemic toxicity is also reduced when vancomycin is applied topically.

Acknowledgement: We are greatly indebted to the pathology department of CMH Kohat and PNS Shifa for timely provision of culture and sensitivity.

Conflict of interest: None.

\section{REFERENCES}

1. Aguiree F, Brown A, Cho NH, Dahlquist G, Dodd S, Dunning T, et al. IDF diabetes atlas. 2013;6(1):29-68.

2. Bonner T, Harvey IS, Sherman L. A Qualitative Inquiry of Lower Extremity Disease Knowledge among African Americans Living With Type 2 Diabetes. Health Promot Pract. 2017;18(6):806-813. doi: $10.1177 / 1524839916688867$.

3. Lauterbach S, Kostev K, Kohlmann T. Prevalence of diabetic foot syndrome and its risk factors in the UK. J Wound Care. 2010;19(8):333-337. doi: 10.12968/ jowc.2010.19.8.77711. 
4. Jung H, Cho J, Kim HK, Kim J, Huh S. Long-term outcomes of infra inguinal bypass surgery for patients with diabetes mellitus and tissue loss. Ann Surg Treat Res. 2015;88(1):3540. doi: 10.4174/astr.2015.88.1.35.

5. Yazdanpanah L, Nasiri M, Adarvishi S. Literature review on the management of diabetic foot ulcer. World J Diabetes. 2015;6(1):37-53. doi:10.4239/wjd.v6.i1.37.

6. Zaitseva EL, Tokmakova AY, Doronina LP, Voronkova IA, Galstyan GR, Shestakova MV, et al. Impact of various wound dressings on wound bed neoangiogenesis in patients with different forms of diabetic foot syndrome. Ter Arkh. 2017;89(10):12-16. doi: 10.17116/terarkh2017891012-16.

7. McIntosh A, Hutchinson A, Home PD, Brown F, Bruce A, Damerell A, et al. Clinical guidelines and evidence review for Type 2 diabetes: management of blood glucose. Sheffield: ScHARR, University of Sheffield. 2001.

8. Garcia EC, Gonzalez RG, Albor AR, Schettino PM. Infections of diabetic foot ulcers with methicillin-resistant Staphylococcus aureus. Int J Low Extrem Wounds. 2015;14(1):44-49. doi: 10.3390/toxins8070209.

9. Ali SM, Basit A, Fawwad A, Ahmedani MY, Miyan Z, Malik RA, et al. Presentation and outcome of diabetic foot at a tertiary care unit. Pak J Med Sci. 2008;24(5):651-656.

10. Shah SF, Hameed S, Khawaja Z, Waqra SH, Zahid MA. Evaluation and Management of Diabetic Foot:A Multicenter study conducted at Rawalpindi, Islamabad. Ann Pak Inst Med Sci. 2011;7(4):233-237.

11. Albaugh KW, Biely SA, Cavorsi JP. The effect of a cellulose dressing and topical vancomycin on methicillin-resistant Staphylococcus aureus (MRSA) and Gram-positive organisms in chronic wounds:a case series. Ostomy Wound Manage. 2013;59(5):34-43.

12. Kavitha KV, Tiwari S, Purandare VB, Khedkar S, Bhosale SS, Unnikrishnan AG, et al. Choice of wound care in diabetic foot ulcer: a practical approach. World J Diabet. 2014;5(4):546-556. doi:10.4239/wjd.v5.i4.546.

13. Ghotaslou R, Memar MY, Alizadeh N. Classification, microbiology and treatment of diabetic foot infections. J Wound Care. 2018;27(7):434-441. doi:10.12968/ jowc.2018.27.7.434.

14. Madanchi N, Malazy OT, Pajouhi M, Heshmat R, Larijani B, Mohajeri MR, et al. Who are diabetic foot patients? A descriptive study on 873 patients. J Diabet Metab Dis. 2013;12:36. doi: 10.1186/2251-6581-12-36.
15. Rubeaan KA, Derwish MA, Ouizi S, Youssef AM, Subhani $\mathrm{SN}$, Ibrahim HM, et al. Diabetic foot complications and their risk factors from a large retrospective cohort study. PLoS One. 2015;10(5):1-17. doi: 10.1371/journal.pone.0124446.

16. Ndosi M, Hughes WA, Brown S, Backhouse M, Lipsky BA, Bhogal M, et al. Prognosis of the infected diabetic foot ulcer:a 12-month prospective observational study. Diabet Med. 2018;35(1):78-88. doi: 10.1111/dme.13537.

17. Amjad SS, Zafar J, Shams N. Bacteriology of diabetic foot in tertiary care hospital; frequency, antibiotic susceptibility and risk factors. J Ayub Med Coll Abbottabad. 2017;29(2):234-240.

18. Miyan Z, Fawwad A, Sabir R, Basit A. Microbiological pattern of diabetic foot infections at a tertiary care center in a developing country. J Pak Med Assoc. 2017;67(5):665-667.

19. Akhi MT, Ghotaslou R, Asgharzadeh M, Varshochi M, Pirzadeh T, Memar MY, et al. Bacterial etiology and antibiotic susceptibility pattern of diabetic foot infections in Tabriz, Iran. GMS Hyg Infect Control. 2015;10 (1):1-6. doi: $10.3205 /$ dgkh000245.

20. Diehr S, Hamp A, Jamieson B, Mendoza M. Clinical inquiries. Do topical antibiotics improve wound healing? J Fam Pract. 2007;56(2):140-144.

21. Meara OSM, Cullum NA, Majid M, Sheldon TA. Systematic review of antimicrobial agents used for chronic wounds. $\mathrm{Br}$ J Surg. 2001;88(1):4-21.

22. SimonsJP, JohnsonJT, Yu VL. The role of topical antimicrobial prophylaxis in patients undergoing contaminated head and neck surgery with flap reconstruction. Laryngoscope. 2001;111(1):329-335.

\section{Authors' Contribution:}

ABS conceived, designed, collected data and did statistical analysis.

SJ, AM, MT did data collection and manuscript writing.

MSA Supervised the whole study \& editing of manuscript. 